\title{
Metaplastic breast carcinoma: a clinical-pathologic study of 97 cases with subset analysis of response to neoadjuvant chemotherapy
}

\author{
Min Han $^{1} \cdot$ Arsalan Salamat $^{2} \cdot$ Li Zhu $^{3} \cdot$ Huina Zhang ${ }^{1} \cdot$ Beth Z. Clark ${ }^{1}$ David J. Dabbs $\mathbb{D}^{1} \cdot$ Gloria J. Carter $^{1}$. \\ Adam M. Brufsky ${ }^{4} \cdot$ Rachel C. Jankowitz ${ }^{4}$ Shannon L. Puhalla ${ }^{4} \cdot$ Ronald R. Johnson ${ }^{2} \cdot$ Atilla Soran $^{2}$. \\ Jennifer G. Steiman ${ }^{2} \cdot$ Priscilla F. McAuliffe $^{2} \cdot$ Emilia J. Diego $^{2} \cdot$ Rohit Bhargava $^{1}$
}

Received: 26 September 2018 / Revised: 15 December 2018 / Accepted: 15 December 2018 / Published online: 5 February 2019

(c) United States \& Canadian Academy of Pathology 2019

\begin{abstract}
Metaplastic breast carcinoma is a rare heterogeneous category of breast cancer, often associated with a poor prognosis. Clinical-pathologic studies with respect to varied morphologic subtypes are lacking. There is also a dearth of studies assessing the response of metaplastic breast carcinoma to neoadjuvant chemotherapy. Cases of metaplastic breast carcinoma diagnosed between 2007 and 2017 were identified. Various clinical-pathologic variables were tested for association with survival. Patients who underwent neoadjuvant chemotherapy were assessed for pathologic response. Median age at diagnosis with metaplastic breast carcinoma was 64 years. With a median follow-up of 39 months, 26 patients (27\%) recurred (24 distant and 2 loco-regional). The overall survival rate of the cohort was $66 \%$ (64/97). A number of variables were associated with survival in univariable analysis; however, in multivariable analysis, only lymph node status and tumor size (pT3 vs. pT1/2) were significantly associated with all survival endpoints: recurrence-free survival, distant recurrence-free survival, overall survival and breast cancer-specific survival. Twenty-nine of $97(30 \%)$ patients with metaplastic breast carcinoma received neoadjuvant chemotherapy. Five (17\%) patients achieved pathologic complete response. Matrix-producing morphology was associated with higher probability of achieving pathologic complete response $(p=0.027)$. Similar to other breast cancer subtypes, tumor size and lymph node status are prognostic in metaplastic carcinomas. The pathologic complete response rate of metaplastic breast carcinoma in our cohort was $17 \%$, higher than previously reported. Although the matrixproducing subtype was associated with pathologic complete response, there was no survival difference with respect to tumor subtypes.
\end{abstract}

These authors contributed equally: Min Han, Arsalan Salamat.

Rohit Bhargava

rbhargava@mail.magee.edu

1 Division of Breast and Gynecologic Pathology, Department of Pathology, University of Pittsburgh School of Medicine, Pittsburgh, PA, USA

2 Division of Surgical Oncology, Department of Surgery, University of Pittsburgh School of Medicine, Pittsburgh, PA, USA

3 Department of Biostatistics, University of Pittsburgh, Pittsburgh, PA, USA

4 Division of Hematology/Oncology, Department of Medicine, University of Pittsburgh School of Medicine, Pittsburgh, PA, USA

\section{Introduction}

Metaplastic breast carcinoma is a rare heterogeneous group of diseases, comprising $0.3-5 \%$ of all breast cancers [1-4]. In 2000, the World Health Organization recognized metaplastic breast carcinoma as a distinct entity. The current classification includes the following histologic variants in the metaplastic carcinoma category: matrix-producing carcinoma, spindle cell carcinoma, squamous cell carcinoma, mixed types of metaplastic carcinoma, low-grade adenosquamous carcinoma, and fibromatosis-like metaplastic carcinoma [5]. Except for the rare low-grade adenosquamous and fibromatosis-like metaplastic carcinomas which are most likely to be identified at an early stage, patients with metaplastic breast carcinoma often presents with higher tumor stage and have a more aggressive clinical course compared with classic ductal and lobular carcinomas of the breast [1, 6-8]. Two recent studies of the National 
Cancer Center Database reported that metaplastic breast carcinomas are the histologic subtype associated with the worst overall survival $[9,10]$.

Surgery ( \pm radiation) is the standard treatment for most metaplastic breast carcinomas. Metaplastic carcinoma is often negative for estrogen receptor (ER) and human epidermal growth factor receptor 2 (HER2), limiting the use of hormonal therapy and HER2 targeted therapy [9]. The use of chemotherapy in metaplastic breast carcinoma is mostly extrapolated from clinical trial results involving typical invasive ductal carcinomas. Published studies regarding response of metaplastic breast carcinomas to chemotherapy, particularly in the neoadjuvant setting, are either old or small in sample size [6, 8]. Despite the traditional notion that metaplastic breast carcinomas are resistant to chemotherapy, systemic chemotherapy is administered to 53.4-73.1\% of patients with metaplastic carcinomas [1, 9].

In recent years, neoadjuvant chemotherapy has been increasingly used as part of the multidisciplinary management of breast cancer [11]. Historically administered to reduce the size of large tumors in order to pursue breastconserving surgery, neoadjuvant chemotherapy is nowadays frequently considered for smaller operable breast cancers. Although neoadjuvant chemotherapy confers no survival benefit over adjuvant chemotherapy, it allows evaluation of tumor response to specific chemotherapy regimens. In addition, pathologic complete response after neoadjuvant chemotherapy has been shown to be an independent predictor for survival compared with cases that fail to achieve pathologic complete response [11-13]. However, there is a dearth of studies evaluating response to neoadjuvant chemotherapy in metaplastic breast carcinoma.

Clinical trials for metaplastic breast carcinomas are hard to achieve due to the rarity of the diagnosis and heterogeneity of its morphologic subtypes. To gain further insight, we performed a retrospective review of metaplastic breast carcinoma diagnosed at a tertiary academic hospital. Approximately one-third of the study cases were treated with neoadjuvant chemotherapy, which allowed assessment of chemo-responsiveness of these tumors. The goals of this study were to identify variables associated with survival and to determine the pathologic complete response rate to neoadjuvant chemotherapy in metaplastic breast carcinoma.

\section{Methods}

A 10-year (2007-2017) electronic data search was performed in the laboratory information system using the keywords "metaplastic carcinoma" plus "breast" in the diagnostic line. Ninety-seven cases met the criteria based on pathology reports and/or review of slides. Any case showing unequivocal metaplastic tumor component was included in the study. Consult cases were excluded. Slides for review were available for 84 cases $(87 \%)$. Two pathologists $(\mathrm{MH}$ and $\mathrm{RB}$ ) estimated the proportion of different tumor components. For patients receiving neoadjuvant chemotherapy, only core biopsy slides were reviewed for sub-classification. The following case information was recorded: patient age, tumor size, nuclear grade and receptor status (semi-quantitative results), lymph node status, loco-regional treatment (surgery and/or radiation), systemic therapy, recurrence-free survival, distant recurrence-free survival, overall survival, and breast cancer-specific survival. For patients not treated with neoadjuvant chemotherapy, the tumor size and nodal status was determined by standard pathology examination of the surgical specimen. For patients treated with neoadjuvant chemotherapy, the radiographic or clinical pretherapy tumor size was taken for staging and the nodal status was considered positive if either metastatic tumor was documented with percutaneous pre-therapy lymph node biopsy or if positive sentinel/axillary node(s) were identified at definitive surgery.

Pathologic complete response was defined as absence of invasive carcinoma in the breast and lack of metastatic carcinoma in the lymph nodes at definitive surgery. Presence of residual ductal carcinoma in-situ was acceptable.

Recurrence-free survival was defined as the time from diagnosis to development of any recurrence (distant or locoregional) or last follow-up. Distant recurrence-free survival was defined as the time from diagnosis to development of distant recurrence or last follow-up. Overall survival duration was defined as the time from diagnosis to death from any cause or last follow-up. Breast cancer-specific survival duration was defined as the time from diagnosis to death due to breast cancer or last follow-up.

Statistical analysis was performed using The $\mathrm{R}$ project for Statistical Computing (https://www.r-project.org/). For continuous variables, the $p$-value was obtained from twosided Wilcoxon rank sum test. For categorical variables, the $p$-value was obtained from the two-sided Fisher's exact test. Patients with missing/unknown information were excluded from the test. Confidence intervals were obtained from Wald normal approximation.

Kaplan-Meier survival curves (for recurrence-free, distant recurrence free, overall and breast cancer-specific survival) were analyzed with respect to clinical-pathologic variables (age, nodal status, tumor size, nuclear grade, ER status, PR status, HER2 status, Ki-67 labeling index, type of surgery [lumpectomy versus mastectomy], administration of radiation, administration of chemotherapy, administration of endocrine therapy, neoadjuvant chemotherapy, tumor subtype [metaplastic only versus admixed], type of most prominent metaplastic component, and number of metaplastic component). $p$-values were obtained using the logrank test. The variables with statistically significant 
associations were included in multivariable Cox proportional hazard model.

\section{Results}

\section{Patient and tumor characteristics}

Patient and tumor characteristics, as well as the therapies received are detailed within Table 1 . Median age at diagnosis was 64 years. Tumors were larger (median size of 3 $\mathrm{cm}$ ) with high nuclear grade (84\%), and most were hormone receptor negative. When positive for hormone receptors, the degree of positivity was low (as assessed by $\mathrm{H}$-scores), and most were $\mathrm{ER}+$ and progesterone receptor (PR) negative. The proportion of metaplastic breast carcinomas which were $\mathrm{ER}-/ \mathrm{PR}+$ was high (8\%). Although most were also HER2 negative, 4 (4\%) were equivocal and $4(4 \%)$ were positive by 2013 American Society of Clinical Oncology/College of American Pathologists HER2 guidelines criteria [14].

Almost one-half of the cases were admixed with carcinoma of no special type. The amount of metaplastic tumor component ranged from 5 to $95 \%$ (median 50\%) in such cases. The most frequent metaplastic subtype in this cohort was matrix producing, followed by squamous and spindle cell. On review, we identified distinct histologic features associated with pure matrix-producing metaplastic carcinoma. Apart from classical chondroid and/or osteoid matrix, we found that matrix-producing metaplastic carcinoma frequently presented as welldemarcated nodule(s) with central necrosis (Fig. 1). Among the cases with slides for review, 29 of 31 (93.5\%) matrix-producing metaplastic breast carcinomas showed large area(s) of de novo necrosis occupying an average of $47 \%$ of the tumor area (range 10-90\%) with a rim of viable cells at the periphery. In some cases, the viable cells were present around blood vessels (peritheliomatous pattern). The tumor cell nuclei in the matrix-producing metaplastic breast carcinoma tend to show variability with smaller (grade 2) nuclei admixed with darker, larger (grade 3) nuclei.

Twenty-nine cases were found to have metastatic carcinoma involving the lymph node(s). Slides of the positive lymph node(s) were available for review in 23 cases. In the involved lymph nodes, 11 tumors showed pure metaplastic morphology (6 squamous, 3 matrix producing and 2 spindle cell), 10 tumors showed mixed no special type and metaplastic components and 2 tumors showed pure no special type component. The metaplastic component(s) present in the lymph nodes resembled the primary tumor in most part. In a subset of the cases, the metaplastic nature of the metastatic tumor was recognizable but the tumor cells showed better differentiation toward no special type compared to the primary tumor.

\section{Patients receiving neoadjuvant chemotherapy}

While most patients in this cohort were treated with surgery, followed by chemotherapy and radiation, twenty-nine (30\%) patients received neoadjuvant chemotherapy. The most commonly administered neoadjuvant chemotherapy regimen was adriamycin, cyclophosphamide, and taxane, used in 16 of $29(55.1 \%)$ patients. Five $(17 \%)$ showed a pathologic complete response (Table 2). Of these 5 cases, 3 were pure matrix producing, 1 mixed matrix-producing and no special type, and one pure spindle cell carcinoma. All tumors resulting in pathologic complete response were ER/ PR negative, one was HER2+ and received targeted neoadjuvant regimen, and all five showed a high Ki-67 labeling index (50\% or higher). One of these five patients recurred (pure matrix producing) and died of disease. The other four were alive and free of disease during the followup period. Interestingly, the patient whose tumor achieved pathologic complete response but who later died was the only patient in the pathologic complete response group who had a positive regional lymph node before therapy. This patient developed distant recurrence 24 months after the diagnosis of cancer and died of disease in 41 months. In univariable analysis, the only variable significantly associated with pathologic complete response was the morphology of the metaplastic tumor component, namely the presence of a matrix-producing component, either pure or admixed with no special type $(p=0.027)$.

In addition to pathologic complete response rate, we also tested the same variables with respect to $>50 \%$ tumor volume reduction in the breast. Seventeen of 29 patients (59\%) showed $>50 \%$ tumor volume reduction in the breast; however, none of the variables were significantly predictive for $>50 \%$ tumor volume reduction.

\section{Survival analysis}

The average follow-up time for the study cohort was 48 months (median 39 months, range: 0-129 months). Twenty-six patients (27\%) recurred (24 distant and 2 locoregional), and one patient had metastatic disease at presentation; with an average disease-free survival of 45 months. The overall survival of the cohort was $66 \%$ (64/97). Of the 33 patients who died, the cause of death was unknown in three cases. Twelve died of non-breast cancer causes and 21 died because of breast cancer with breast cancer-specific survival rate of $78 \%$.

In univariable analysis, negative nodal status $(p<0.001$, $p<0.001)$, lumpectomy as primary surgery $(p=0.029, p=$ $0.028)$, single metaplastic tumor component $(p=0.006$, 
Table 1 Patient and tumor characteristics and therapies received

\begin{tabular}{|c|c|c|}
\hline & $N$ & $\%$ \\
\hline \multicolumn{3}{|l|}{ Age group } \\
\hline$\leq 55$ & 33 & 34 \\
\hline$>55$ & 64 & 66 \\
\hline Median age (inter-quartile range) & $64(51-75)$ & - \\
\hline \multicolumn{3}{|l|}{$\mathrm{pT}$} \\
\hline pT1 & 25 & 26 \\
\hline pT2 & 43 & 44 \\
\hline $\mathrm{pT} 3 / 4$ & 27 & 28 \\
\hline Unknown & 2 & 2 \\
\hline \multicolumn{3}{|l|}{ Nuclear grade } \\
\hline G1 & 0 & 0 \\
\hline G2 & 4 & 4 \\
\hline G3 & 81 & 84 \\
\hline Unknown & 12 & 12 \\
\hline \multicolumn{3}{|l|}{ Estrogen Receptor (ER) } \\
\hline Positive & 19 & 20 \\
\hline Negative & 78 & 80 \\
\hline $\mathrm{H}$-score range for positive & $1-150$ & - \\
\hline Median (IQR) for positive & $10(10-50)$ & - \\
\hline \multicolumn{3}{|l|}{ Progesterone receptor (PR) } \\
\hline Positive & 11 & 11 \\
\hline Negative & 86 & 89 \\
\hline $\mathrm{H}$-score range for positive & $1-140$ & - \\
\hline Median (IQR) for positive & $6(3-20)$ & - \\
\hline \multicolumn{3}{|l|}{ HER2 } \\
\hline Positive & 4 & 4 \\
\hline Equivocal & 4 & 4 \\
\hline Negative & 89 & 92 \\
\hline \multicolumn{3}{|l|}{$\mathrm{Ki}-67$} \\
\hline$<50 \%$ & 17 & 17 \\
\hline$\geq 50 \%$ & 55 & 57 \\
\hline Unknown & 25 & 26 \\
\hline Median (inter-quartile range) & $65(50-80)$ & - \\
\hline \multicolumn{3}{|l|}{ Receptor profile } \\
\hline $\mathrm{ER}+/ \mathrm{PR}+$ & 3 & 3 \\
\hline $\mathrm{ER}-/ \mathrm{PR}-$ & 70 & 72 \\
\hline $\mathrm{ER}+/ \mathrm{PR}-$ & 16 & 17 \\
\hline $\mathrm{ER}-/ \mathrm{PR}+$ & 8 & 8 \\
\hline $\mathrm{ER}+/ \mathrm{HER} 2+$ & 2 & 2 \\
\hline ER -/HER 2- & 76 & 78 \\
\hline $\mathrm{ER}+/ \mathrm{HER} 2-$ & 17 & 18 \\
\hline ER-/HER2+ & 2 & 2 \\
\hline \multicolumn{3}{|l|}{$\mathrm{pN}$} \\
\hline pNO & 62 & 64 \\
\hline $\mathrm{pN} 1$ & 21 & 22 \\
\hline $\mathrm{pN} 2$ & 3 & 3 \\
\hline
\end{tabular}

Table 1 (continued)

\begin{tabular}{lll}
\hline & $N$ & $\%$ \\
\hline pN3 & 5 & 5 \\
Unknown & 6 & 6 \\
Tumor subtype & & \\
Admixed with NST & 49 & 51 \\
Metaplastic only & 48 & 49 \\
Most prominent tumor component overall & & \\
Matrix producing & 31 & 32 \\
NST & 24 & 25 \\
Squamous & 21 & 22 \\
Spindle & 18 & 18 \\
Other & 3 & 3
\end{tabular}

Most prominent metaplastic component when not admixed with NST (48 cases)

$\begin{array}{lll}\text { Matrix producing } & 19 & 40 \\ \text { Squamous } & 15 & 31 \\ \text { Spindle } & 14 & 29\end{array}$

Local treatment

Lumpectomy + radiation $\quad 31 \quad 32$

$\begin{array}{lll}\text { Lumpectomy, no radiation } & 9 & 9\end{array}$

Mastectomy + radiation $\quad 15 \quad 16$

Mastectomy, no radiation $\quad 40 \quad 41$

Mastectomy, radiation scheduled $\quad 1 \quad 1$

No primary surgery $\quad 1 \quad 1$

Systemic therapy

$\begin{array}{lll}\text { Chemotherapy only } & 57 & 59\end{array}$

Chemo + endocrine therapy $\quad 6 \quad 6$

Endocrine therapy only 3

Neither chemo nor endocrine therapy $\quad 31 \quad 32$

Neoadjuvant chemotherapy

$\begin{array}{lll}\text { Yes } & 29 & 30 \\ \text { No } & 68 & 70\end{array}$

$p T$ pathologic tumor stage, $p N$ pathologic nodal stage, $N S T$ carcinoma of no special type

$p=0.004)$, and smaller tumor size (pT1/2 rather than $\mathrm{pT} 3$, $p=0.001, \quad p<0.001)$ were associated with improved recurrence-free and distant recurrence-free survival. For overall survival, negative nodal status $(p<0.001)$, administration of radiation $(p=0.042)$ and chemotherapy $(p=$ $0.003)$, and smaller tumor size $(p<0.001)$ were associated with better survival. For breast cancer-specific survival, negative nodal status $(p<0.001)$, lumpectomy as primary surgery $(p=0.039)$, and smaller tumor size $(p<0.001)$ were associated with better survival.

In multivariable analysis, lymph node status and tumor size (pT3 vs. pT1/2) were the only two variables that were associated with all survival endpoints, recurrence-free, distant recurrence free, overall, and breast cancer-specific 

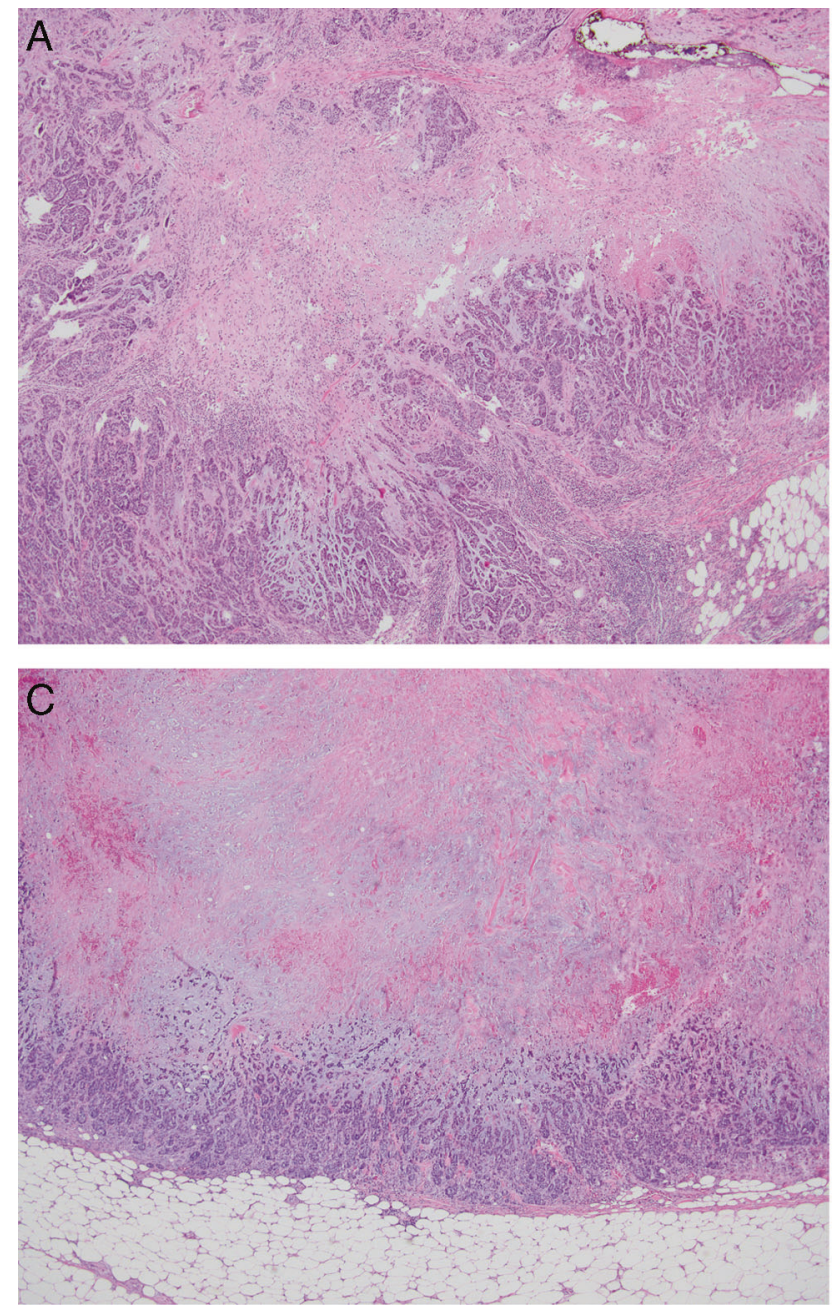

Fig. 1 Low-power view of representative cases showing metaplastic breast carcinoma with well-demarcated nodule(s) with central necrosis

survival (Figs. 2 and 3, Table 3). More than one metaplastic component was associated with worse recurrence-free survival (hazard ratio of $7.21, p$-value: $0.001,95 \%$ CI: 2.16-24.10) and breast cancer-specific survival (hazard ratio: 5.42, $p$-value: $0.038,95 \% \mathrm{CI}: 1.10-26.60)$. Administration of radiation therapy and chemotherapy were both associated with improved overall survival (hazard ratio of 0.300 for radiation, $p$-value: $0.041,95 \%$ CI: $0.095-0.950$; hazard ratio of 0.397 for chemotherapy, $p$-value: 0.039 , 95\% CI: 0.165-0.954) without a significant effect on recurrence-free survival (Table 3).

\section{Discussion}

We identified 97 metaplastic breast carcinomas diagnosed and treated at our institution in the past 10 years (between 2007 and 2017). This number was much higher than the reported 27 cases over 21 years (between 1976 and 1997) in
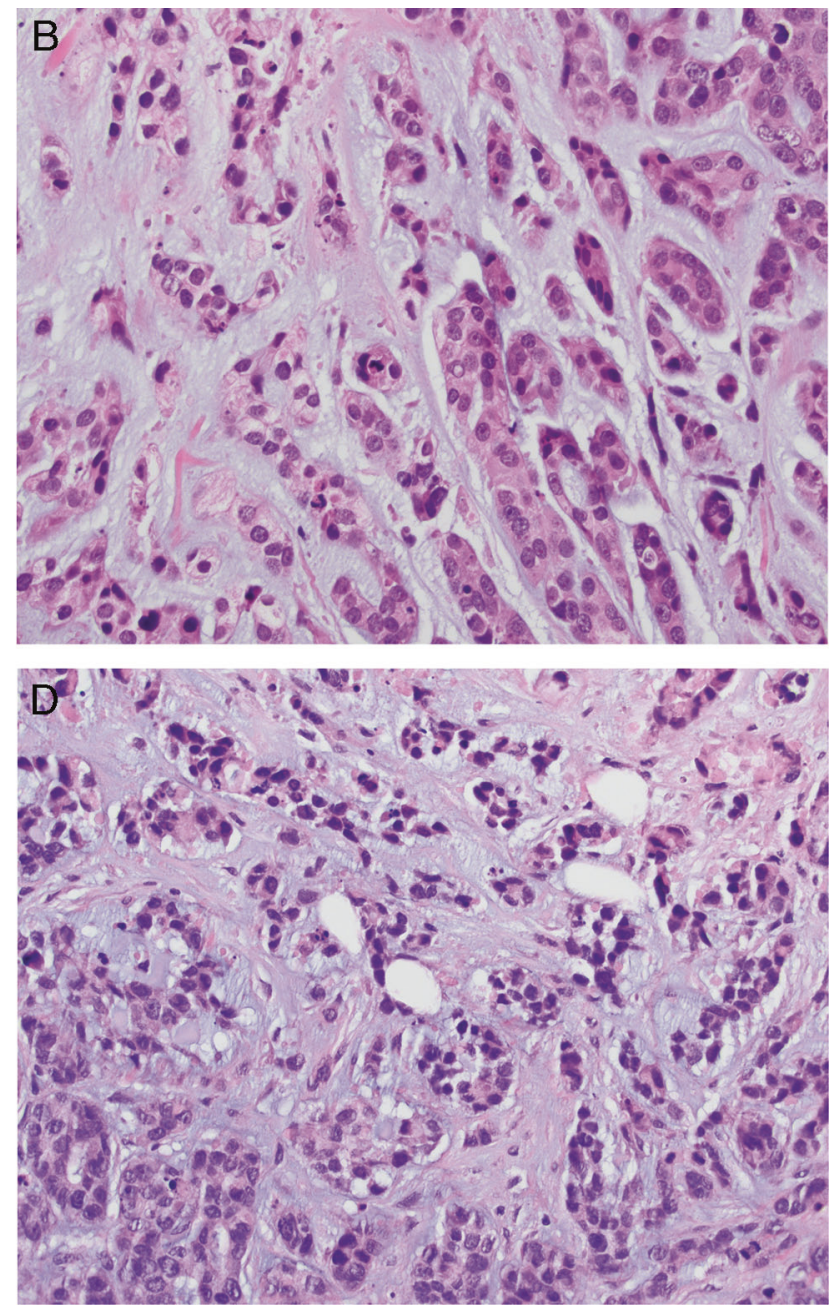

(a, c). High-power view of the same cases, showing abundant chondroid matrix with moderate to marked nuclear pleomorphism $(\mathbf{b}, \mathbf{d})$

a previous Mayo clinic study [8], reflecting the increased awareness of this specific category of breast cancer after the World Health Organization recognition in 2000 [5]. The patients in our cohort were slightly older at diagnosis (median age 64 years) than the typical age at presentation for breast cancer. Previous literature has shown significantly worse recurrence-free and overall survival associated with metaplastic breast carcinoma than with classic triplenegative breast cancer [9, 15-17]. Our study did not include classic breast cancers, but our results showed that metaplastic breast carcinoma patients had an overall survival rate of $66 \%$ (with 39 months median follow-up), worse than published data about classic triple-negative breast cancer [10]. Squamous and spindle cell differentiation have been shown to be associated with worse prognosis than other metaplastic components [16]. Our study did not reveal any significant difference in overall or recurrence-free survival among different metaplastic breast carcinoma subtypes. However, our results showed that the presence of 
Table 2 Association of clinical and pathologic variables with pathologic complete response (pCR) to neoadjuvant chemotherapy

\begin{tabular}{|c|c|c|c|c|}
\hline & Total $(N=29)$ & $\begin{array}{l}\text { Residual tumor/negative } \\
\text { for pCR }(N=24,83 \%)\end{array}$ & $\mathrm{pCR}(N=5,17 \%)$ & $p$-value \\
\hline \multicolumn{5}{|c|}{ Continuous variables: mean (SD) } \\
\hline Age & $54.9(13.3)$ & $54.5(14.2)$ & $57.0(8.6)$ & 0.58 \\
\hline Tumor size & $4.6(3.2)$ & $5.0(3.4)$ & $2.6(0.8)$ & 0.13 \\
\hline Ki-67 labeling index (\%) & 70.8 (18.2), 5 unknown & 72.3 (18.7), 4 unknown & 63.8 (16.0), 1 unknown & 0.27 \\
\hline NST\% & 32 (37.8), 4 unknown & 36.5 (38.6), 4 unknown & $14.0(31.3)$ & 0.17 \\
\hline \multicolumn{5}{|l|}{ Categorical variables: $N(\%)$} \\
\hline \multicolumn{5}{|l|}{ Age } \\
\hline$\leq 55$ & $16(55 \%)$ & $15(62 \%)$ & $1(20 \%)$ & \multirow[t]{2}{*}{0.14} \\
\hline$>55$ & $13(45 \%)$ & $9(38 \%)$ & $4(80 \%)$ & \\
\hline \multicolumn{5}{|l|}{ pT } \\
\hline pT1 & $6(21 \%)$ & $4(17 \%)$ & $2(40 \%)$ & \multirow[t]{3}{*}{0.18} \\
\hline pT2 & $13(45 \%)$ & $10(42 \%)$ & $3(60 \%)$ & \\
\hline pT3 & $10(34 \%)$ & $10(42 \%)$ & 0 & \\
\hline \multicolumn{5}{|l|}{ ER } \\
\hline Pos & $6(21 \%)$ & $6(25 \%)$ & 0 & \multirow[t]{2}{*}{0.56} \\
\hline Neg & $23(79 \%)$ & $18(75 \%)$ & $5(100 \%)$ & \\
\hline \multicolumn{5}{|l|}{ PR } \\
\hline Pos & 0 & 0 & 0 & \multirow[t]{2}{*}{ NA } \\
\hline Neg & $29(100 \%)$ & $24(100 \%)$ & $5(100 \%)$ & \\
\hline \multicolumn{5}{|l|}{ HER2 } \\
\hline Positive & $2(7 \%)$ & $1(4 \%)$ & $1(20 \%)$ & \multirow[t]{3}{*}{0.45} \\
\hline Equivocal & $1(3 \%)$ & $1(4 \%)$ & 0 & \\
\hline Negative & $26(90 \%)$ & $22(92 \%)$ & $4(80 \%)$ & \\
\hline \multicolumn{5}{|l|}{ KI67 } \\
\hline$<50$ & $2(8 \%)$ & $2(10 \%)$ & 0 & \multirow[t]{2}{*}{1} \\
\hline$\geq 50$ & $22(92 \%), 5$ unknown & $18(90 \%), 4$ unknown & $4(100 \%), 1$ unknown & \\
\hline \multicolumn{5}{|l|}{ Admixed with NST } \\
\hline Yes & $15(52 \%)$ & $14(58 \%)$ & $1(20 \%)$ & \multirow[t]{2}{*}{0.17} \\
\hline No & $14(48 \%)$ & $10(42 \%)$ & $4(80 \%)$ & \\
\hline \multicolumn{5}{|c|}{ Most prominent metaplastic component } \\
\hline Matrix producing & $17(59 \%)$ & $13(54 \%)$ & $4(80 \%)$ & \multirow[t]{3}{*}{$0.03 *$} \\
\hline Spindle & $1(3 \%)$ & 0 & $1(20 \%)$ & \\
\hline Squamous & $11(38 \%)$ & $11(46 \%)$ & 0 & \\
\hline \multicolumn{5}{|l|}{ NST\% } \\
\hline$\leq 10 \%$ & $13(52 \%)$ & $9(45 \%)$ & $4(80 \%)$ & \multirow[t]{4}{*}{0.60} \\
\hline$>10 \%, \leq 50 \%$ & $3(12 \%)$ & $3(15 \%)$ & 0 & \\
\hline$>50 \%, \leq 90 \%$ & $8(32 \%)$ & $7(35 \%)$ & $1(20 \%)$ & \\
\hline$>90 \%$ & $1(4 \%), 4$ unknown & $1(5 \%), 4$ unknown & 0 & \\
\hline
\end{tabular}

$N S T$ carcinoma of no special type, $p T$ pathologic tumor stage, $E R$ estrogen receptor, $P R$ progesterone receptor, $N A$ not applicable

*Statistically significant. Wilcoxon rank sum test was used for continuous variables, and Fisher's exact test was used for categorical variables. Missing values were removed for test. $p$-values are rounded up to 2 decimal points

more than one metaplastic component was associated with worse recurrence-free and breast cancer-specific survival. This is plausible because increased plasticity, a stem-celllike feature, often implies poor differentiation. Similar to typical breast carcinomas, our results indicated that negative lymph node status and smaller tumor size were predictors for better recurrence-free and breast cancer-specific survival in metaplastic breast carcinoma. Nevertheless, caution is urged with respect to survival analysis, given that the patients were not treated uniformly as part of a clinical trial. 

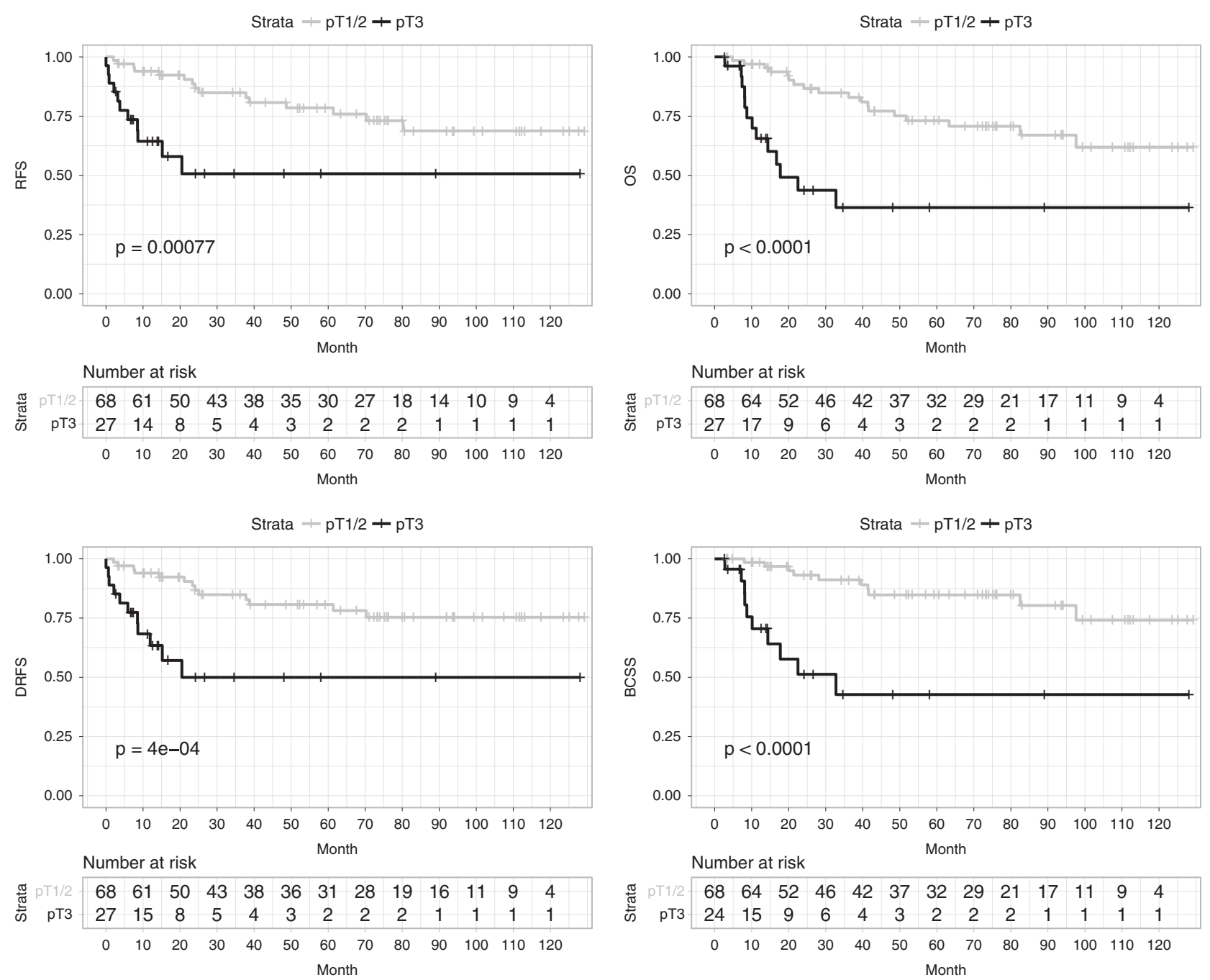

Fig. 2 Association of tumor size with survival

In this study, we reported the largest number (29 of 97) of patients with metaplastic breast carcinoma undergoing neoadjuvant chemotherapy at a single institution and the highest rate of pathologic complete response (17.2\%) for metaplastic breast carcinoma. In a previous MD Anderson study, only $2(10 \%)$ patients with metaplastic breast carcinoma treated with neoadjuvant chemotherapy achieved pathologic complete response [6]. A recent Johns Hopkins study reported 13\% (6 of 45) of patients diagnosed with metaplastic breast carcinoma received neoadjuvant chemotherapy, among whom only 1 patient achieved pathologic complete response [18]. None of the patients with metaplastic breast carcinoma who received neoadjuvant chemotherapy in a recent Turkish study $(n=8)$ [15] or in a Japanese study $(n=14)$ [17] achieved pathologic complete response. In a recent review of the National Cancer Database from 2010 to 2014, 33,162 patients with breast cancers underwent neoadjuvant chemotherapy, of whom $19.2 \%$ of patients achieved pathologic complete response [11]. Although National Cancer Database study applied slightly more strict criteria for pathologic complete response by excluding cases with ductal carcinoma in-situ in the resection specimen, the overall pathologic complete response rate in our study is similar to the pathologic complete response rate reported in the National Cancer Database study [11].

Although metaplastic breast carcinoma is often regarded as having a triple-negative phenotype, they actually show various immunoprofiles. Only $70.3 \%$ of the 2451 metaplastic breast carcinoma cases in the National Cancer Database study were truly negative for ER, PR, and HER2; HER2 was positive in 118 (4.8\%) [10]. Our study showed similar results, with $67 \%$ (65 of 97) of metaplastic breast carcinoma being triple negative. Weakly to moderately ER positive metaplastic breast carcinoma constituted $20 \%$ of our cases. Interestingly, the ER-/PR+ group represented a 

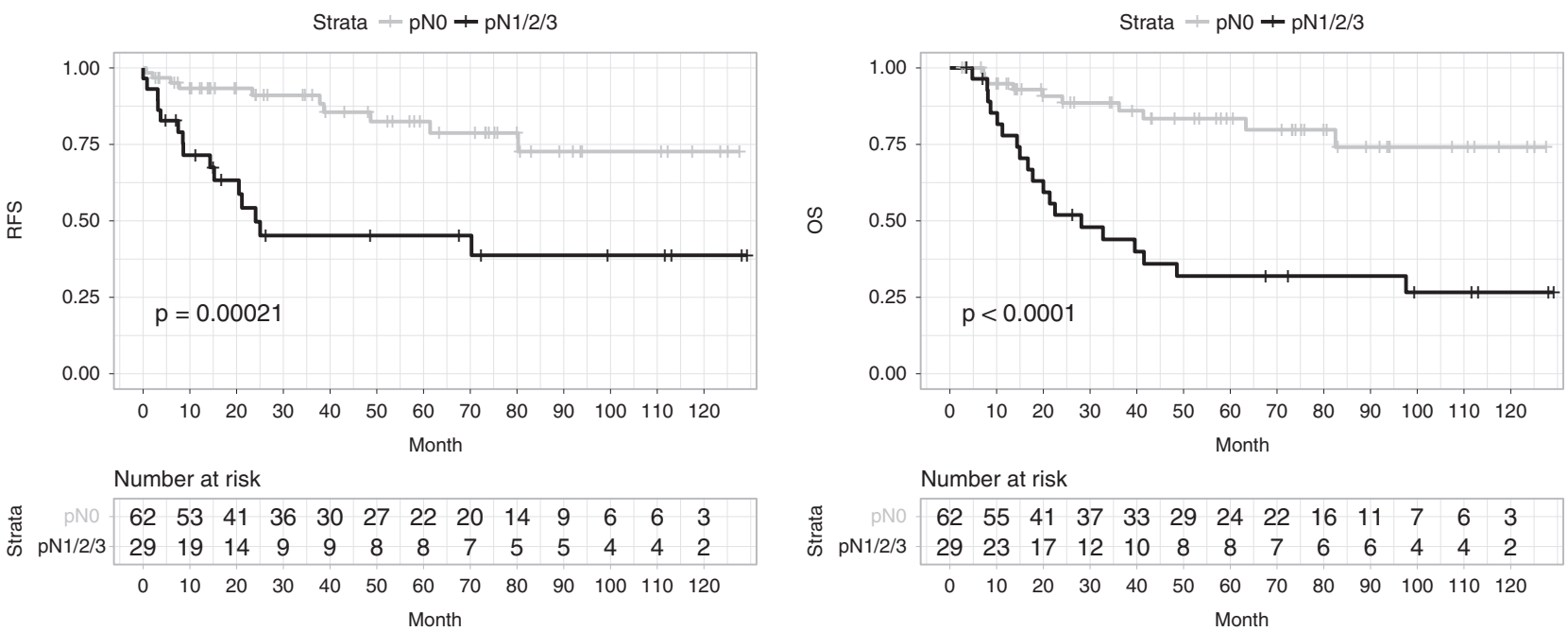

Number at risk
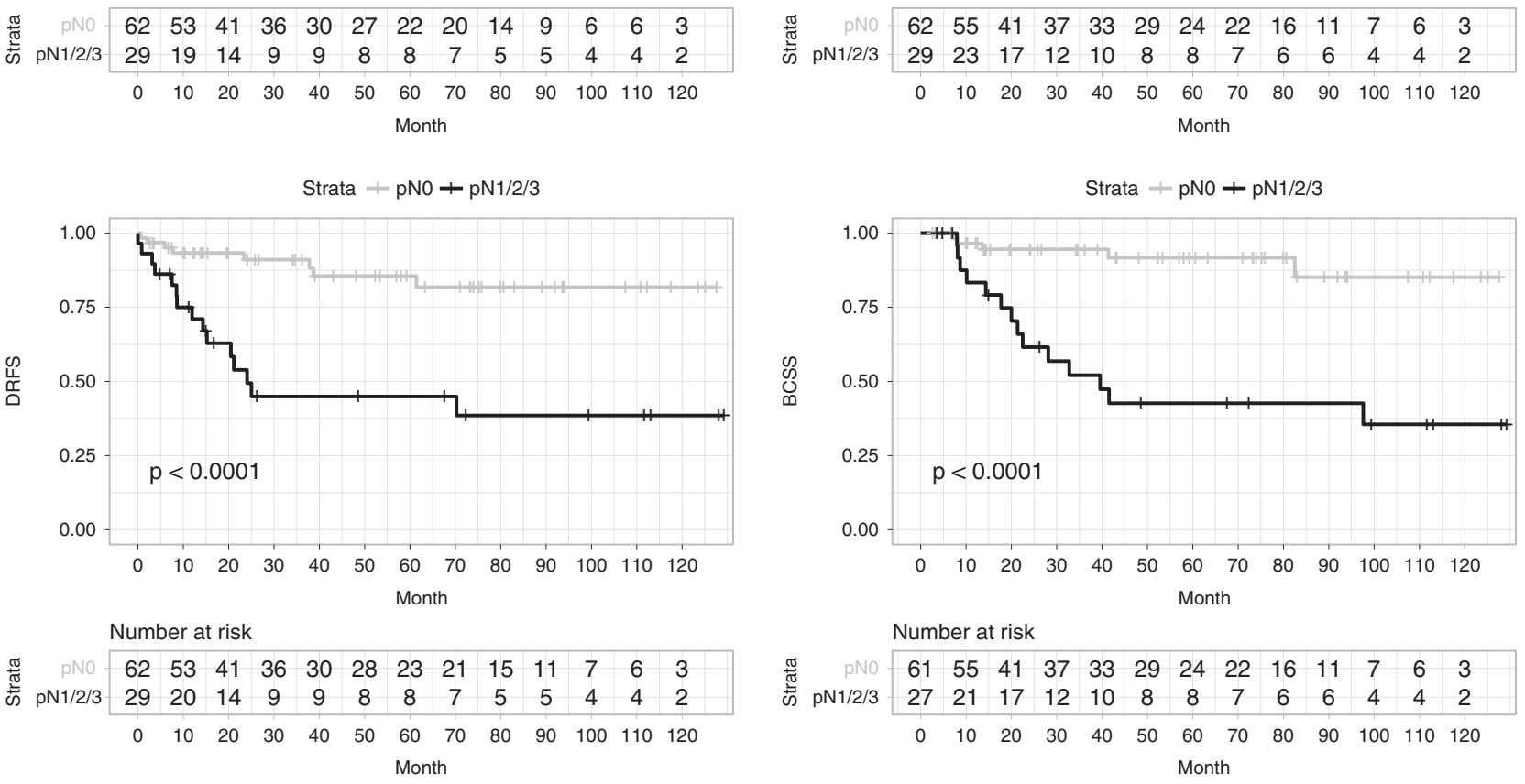

Fig. 3 Association of lymph node status with survival

higher number of cases than we expected (8\%). Wright and colleagues studied the influence of hormonal status on the prognosis of metaplastic breast carcinoma using the Surveillance, Epidemiology, and End Results data from 2000 to 2010 [19]. The authors showed that 79\% (1632 of 2066) of metaplastic breast carcinomas in the Surveillance, Epidemiology, and End Results database were negative for both $\mathrm{ER}$ and PR. The rest of the cases were $\mathrm{ER}+\mathrm{PR}+(8.8 \%)$, $\mathrm{ER}+/ \mathrm{PR}-(7.6 \%)$ and $\mathrm{ER}-/ \mathrm{PR}+(4.6 \%)$. The authors concluded that hormonal status does not affect the 5-year overall survival (65.7 vs. $63.5 \%, p=0.70$ ) [19]. However, the author did acknowledge that the lack of information about the anti-hormonal therapy and the HER2 status of the cases was a significant drawback of the study. The response of ER-positive metaplastic breast carcinoma to antihormonal therapy is largely unknown. In fact, not all hormone receptor-positive metaplastic breast carcinoma in the National Cancer Database study received hormonal therapy;
[9] $72(11.3 \%)$ patients with known hormonal receptor status showed ER positivity but only 57 (6.4\%) patients received hormonal therapy [9]. The authors did not comment on tumor response to hormonal therapy, which may be of interest in future studies. In our current study, 6 of the 19 $\mathrm{ER}+$ patients received endocrine therapy. ER + status was associated with worse distant recurrence-free and breast cancer-specific survival (Table 3), but the administration of endocrine therapy did not have any significant impact on survival. However, it is to be noted that the total number of $\mathrm{ER}+$ patients in the current study is rather small and the impact of ER status on survival should be confirmed using larger datasets.

HER2 positivity is even rarer and no study evaluating the effectiveness of anti-HER2 therapy in metaplastic breast carcinoma is available. In the current study, HER2 was positive (immunohistochemical score of $3+$ ) in $4(4.1 \%)$ cases, including 2 squamous cell, 1 spindle cell, and 1 
Table 3 Variables significant for survival in multivariable analysis

\begin{tabular}{|c|c|c|c|c|}
\hline Variables & RFS & DRFS & OS & BCSS \\
\hline \multicolumn{5}{|c|}{ Lymph node positive tumors } \\
\hline Hazard ratio & 4.030 & 5.150 & 4.650 & 8.380 \\
\hline$p$-value & 0.002 & 0.001 & $<0.001$ & 0.001 \\
\hline $95 \% \mathrm{CI}$ & $1.660-9.780$ & $1.960-13.500$ & $2.030-10.600$ & $2.520-27.800$ \\
\hline \multicolumn{5}{|c|}{ Tumor size (pT3 tumors) } \\
\hline Hazard ratio & 5.0 & 5.920 & 5.700 & 11.500 \\
\hline$p$-value & 0.001 & 0.001 & $<0.001$ & $<0.001$ \\
\hline $95 \% \mathrm{CI}$ & $1.880-13.300$ & $2.080-16.800$ & $2.210-14.700$ & $3.350-39.800$ \\
\hline \multicolumn{5}{|c|}{ Metaplastic component > 1} \\
\hline Hazard ratio & 7.210 & 8.120 & Not significant & 5.420 \\
\hline$p$-value & 0.001 & 0.001 & & 0.038 \\
\hline $95 \% \mathrm{CI}$ & $2.160-24.100$ & $2.270-29.100$ & & $1.100-26.600$ \\
\hline \multicolumn{5}{|c|}{ Estrogen receptor-positive tumors } \\
\hline Hazard ratio & Not significant & 3.260 & Not significant & 5.640 \\
\hline$p$-value & & 0.043 & & 0.022 \\
\hline $95 \% \mathrm{CI}$ & & $1.040-10.200$ & & $1.280-24.900$ \\
\hline \multicolumn{5}{|c|}{ Administration of radiation } \\
\hline Hazard ratio & Not significant & Not significant & 0.300 & 0.203 \\
\hline$p$-value & & & 0.041 & 0.045 \\
\hline $95 \% \mathrm{CI}$ & & & $0.095-0.950$ & $0.043-0.962$ \\
\hline \multicolumn{5}{|c|}{ Administration of chemotherapy } \\
\hline Hazard ratio & Not significant & Not significant & 0.397 & Not significant \\
\hline$p$-value & & & 0.039 & \\
\hline $95 \% \mathrm{CI}$ & & & $0.165-0.954$ & \\
\hline
\end{tabular}

$C I$ confidence interval, RFS recurrence-free survival, DRFS distant recurrence-free survival, $O S$ overall survival, BCSS breast cancer-specific survival

matrix-producing type of metaplastic breast carcinoma. HER2 positivity was identified in the metaplastic tumor component. An additional four cases showed equivocal HER2 results (IHC score $2+$ and fluorescence in-situ hybridization [FISH] equivocal by 2013 HER2 guideline criteria). Three of the four HER2 positive metaplastic breast carcinoma patients in our study received targeted therapy, two in the neoadjuvant setting and another one post surgery. One of the two neoadjuvant patients receiving trastuzumabbased chemotherapy achieved pathologic complete response. Regardless of pathologic complete response or administration of targeted therapy, all four HER $2+$ patients were alive during follow-up period. It is difficult to assess the effect of targeted therapy in this anecdotal experience.

Metaplastic breast carcinoma has been shown to be extremely heterogeneous in morphology and in molecular analysis [3, 20]. Matrix-producing type metaplastic breast carcinoma was the most common type of metaplastic carcinoma in our study. Interestingly, the matrix producing variant was also the only histo-morphologic feature associated with higher probability of achieving pathologic complete response $(p<0.05)$. This most likely represents the biological differences among different subtypes of metaplastic breast carcinoma and their different sensitivity to the commonly used chemotherapeutic regimens. However, this finding should be further confirmed in larger multi-institutional studies. Nevertheless, it is important to accurately diagnose/ recognize matrix-producing metaplastic breast carcinoma, which is characterized by abundant myxoid or chondroid stroma with a large area of central necrosis and a rim of viable tumor cells with moderate to severe nuclear pleomorphism. Although matrix-producing subtype was predictive of a higher likelihood of achieving pathologic complete response in this cohort, no survival benefit was associated with either pathologic complete response status or matrix-producing type histology. The literature regarding metaplastic breast carcinoma histologic sub-types and pathologic complete response is limited; only a case report about pathologic complete response in primary breast squamous cell carcinoma is available [21]. None of the 11 metaplastic breast carcinoma with squamous differentiation in the current study achieved pathologic complete response after neoadjuvant chemotherapy. Given the limited data in this study and in the literature, caution is urged in over-interpretation of the associations of 
the morphologic features and achieving a pathologic complete response.

The pathologic complete response rate of metaplastic breast carcinoma in our cohort was $17.2 \%$, higher than previously reported. Matrix-producing morphology was associated with a higher probability of achieving pathologic complete response. However, this did not translate into survival advantage. In addition to positive lymph node status and larger tumor size (pT3), the presence of more than one metaplastic component was an adverse factor for recurrence-free and breast cancer-specific survival in metaplastic breast carcinoma. Additional large multiinstitutional prospective studies with central pathology review are needed to definitively identify prognostic and predictive factors in metaplastic breast carcinoma.

\section{Compliance with ethical standards}

Conflict of interest The authors declare that they have no conflict of interest.

Publisher's note: Springer Nature remains neutral with regard to jurisdictional claims in published maps and institutional affiliations.

\section{References}

1. Pezzi CM, Patel-Parekh L, Cole K, Franko J, Klimberg VS, Bland K. Characteristics and treatment of metaplastic breast cancer: analysis of 892 cases from the National Cancer Data Base. Ann Surg Oncol. 2007;14:166-73.

2. Weigelt B, Eberle C, Cowell CF, Ng CK, Reis-Filho JS. Metaplastic breast carcinoma: more than a special type. Nat Rev Cancer. 2014;14:147-8.

3. Weigelt B, Ng CK, Shen R, Popova T, Schizas M, Natrajan R, et al. Metaplastic breast carcinomas display genomic and transcriptomic heterogeneity [corrected]. Mod Pathol. 2015;28:340-51.

4. Gibson GR, Qian D, Ku JK, Lai LL. Metaplastic breast cancer: clinical features and outcomes. Am Surg. 2005;71:725-30.

5. Lakhani SR, Ellis IO, Schnitt SJ, editors. WHO classsification of tumours of the breast. Lyon: IARC Press; 2012.

6. Hennessy BT, Giordano S, Broglio K, Duan Z, Trent J, Buchholz TA, et al. Biphasic metaplastic sarcomatoid carcinoma of the breast. Ann Oncol. 2006;17:605-13.

7. Park HS, Park S, Kim JH, Lee JH, Choi SY, Park BW, et al. Clinicopathologic features and outcomes of metaplastic breast carcinoma: comparison with invasive ductal carcinoma of the breast. Yonsei Med J. 2010;51:864-9.

8. Rayson D, Adjei AA, Suman VJ, Wold LE, Ingle JN. Metaplastic breast cancer: prognosis and response to systemic therapy. Ann Oncol. 1999;10:413-9.

9. Mills MN, Yang GQ, Oliver DE, Liveringhouse CL, Ahmed KA, Orman AG, et al. Histologic heterogeneity of triple negative breast cancer: a National Cancer Centre Database analysis. Eur J Cancer. 2018;98:48-58.

10. Ong CT, Campbell BM, Thomas SM, Greenup RA, Plichta JK, Rosenberger LH, et al. Metaplastic breast cancer treatment and outcomes in 2500 patients: a retrospective analysis of a National Oncology Database. Ann Surg Oncol. 2018;25:2249-60.

11. Fayanju OM, Ren Y, Thomas SM, Greenup RA, Plichta JK, Rosenberger $\mathrm{LH}$, et al. The clinical significance of breast-only and node-only pathologic complete response (pCR) after neoadjuvant chemotherapy (NACT): a review of 20,000 breast cancer patients in the National Cancer Data Base (NCDB). Ann Surg. 2018;268:591-601.

12. Bonnefoi H, Litiere S, Piccart M, MacGrogan G, Fumoleau P, Brain E, et al. Pathological complete response after neoadjuvant chemotherapy is an independent predictive factor irrespective of simplified breast cancer intrinsic subtypes: a landmark and twostep approach analyses from the EORTC 10994/BIG 1-00 phase III trial. Ann Oncol. 2014;25:1128-36.

13. Cortazar P, Zhang L, Untch M, Mehta K, Costantino JP, Wolmark $\mathrm{N}$, et al. Pathological complete response and long-term clinical benefit in breast cancer: the CTNeoBC pooled analysis. Lancet. 2014;384:164-72.

14. Wolff AC, Hammond ME, Hicks DG, Dowsett M, McShane $\mathrm{LM}$, Allison $\mathrm{KH}$, et al. Recommendations for human epidermal growth factor receptor 2 testing in breast cancer: American Society of Clinical Oncology/College of American Pathologists clinical practice guideline update. J Clin Oncol. 2013; 31:3997-4013.

15. Aydiner A, Sen F, Tambas M, Ciftci R, Eralp Y, Saip P, et al. Metaplastic breast carcinoma versus triple-negative breast cancer: survival and response to treatment. Medicine. 2015;94:e2341.

16. Lee H, Jung SY, Ro JY, Kwon Y, Sohn JH, Park IH, et al. Metaplastic breast cancer: clinicopathological features and its prognosis. J Clin Pathol. 2012;65:441-6.

17. Nagao T, Kinoshita T, Hojo T, Tsuda H, Tamura K, Fujiwara Y. The differences in the histological types of breast cancer and the response to neoadjuvant chemotherapy: the relationship between the outcome and the clinicopathological characteristics. Breast. 2012;21:289-95.

18. Cimino-Mathews A, Verma S, Figueroa-Magalhaes MC, Jeter SC, Zhang Z, Argani P, et al. A clinicopathologic analysis of 45 patients with metaplastic breast carcinoma. Am J Clin Pathol. 2016;145:365-72.

19. Paul Wright G, Davis AT, Koehler TJ, Melnik MK, Chung MH. Hormone receptor status does not affect prognosis in metaplastic breast cancer: a population-based analysis with comparison to infiltrating ductal and lobular carcinomas. Ann Surg Oncol. 2014;21:3497-503.

20. Lehmann BD, Bauer JA, Chen X, Sanders ME, Chakravarthy AB, Shyr $Y$, et al. Identification of human triple-negative breast cancer subtypes and preclinical models for selection of targeted therapies. J Clin Invest. 2011;121:2750-67.

21. Alan O, Telli TA, Ercelep O, Hasanov R, Simsek ET, Mutis A, et al. A case of primary squamous cell carcinoma of the breast with pathologic complete response after neoadjuvant chemotherapy. Curr Probl Cancer. 2018 May 9. pii: S0147-0272(17)30212X. https://doi.org/10.1016/j.currproblcancer.2018.04.003. [Epub ahead of print]. PMID: 29880396. 